\title{
Etnocompetitividad textil, tendencia histórica que marca el futuro empresarial.
}

Etnocompetitividad textiles, historical trend that marks the future of business.

Cobo Carrasco Paul Alejandro. ${ }^{1}$, Mancheno Saá Juan Patricio. ${ }^{2}$, Guamán Llamuca Carlos Alberto. ${ }^{3}$ \& Mancheno Saá Marcelo Javier. ${ }^{4}$

Recibido: 15-03-2017 / Revisado: 17-05-2017 Aceptado: 15-06-2018/ Publicado: 01-07-2018

\begin{abstract}
.
DOI: https://doi.org/10.33262/cienciadigital.v2i3.160

Ecuador is a pluri-ethical and multicultural country where the ancestral distinctive signs are implicit in the actions of the people, with the maturity of the people it becomes increasingly evident a target that is not ashamed of its roots but rather wants to make them explicit. The Ethnocompetitivity directed to ancestral textiles is a new trend that takes force in the last epoch. The work developed is based on two sources of analysis: 1) A literary analysis of the topic, 2) Surveys based on descriptive statistics in the general and specific market environment. The aim is to establish the perception of the people regarding the subject. Making this study an application of descriptive statistics to generate statistical guidelines rather than industry parameters
\end{abstract}

Keywords: Ethno Competitiveness, Ancestral, Multiculturalism, Culture

\section{Resumen.}

El Ecuador es un país pluri ético y multicultural donde los signos distintivos ancestrales están implícitos en el actuar del pueblo, con la madurez en las personas se hace cada vez más evidente un target que no se avergüenza de sus raíces sino que más bien quiere hacerlas explicitas. La Etnocompetitividad direccionada a textiles ancestrales es una nueva tendencia que adopta fuerza en la última época. El trabajo desarrollado se basa en dos fuentes de análisis: 1) Un análisis literario del tema, 2)

\footnotetext{
${ }^{1}$ Universidad Técnica de Ambato, FADCAM, Ambato, Ecuador, lex_1710@live.com

${ }^{2}$ Pontificia Universidad Católica del Ecuador sede Ambato, de la Escuela de Administración de Empresas EAE,el correo es juan-pa007@hotmail.com

${ }^{3}$ Universidad Técnica de Ambato, FADCAM, Ambato, Ecuador, caguaman@uta.edu.ec

${ }^{4}$ Universidad Técnica de Ambato, FADCAM, Ambato, Ecuador, mj.mancheno@uta.edu.ec
} 
Encuestas basadas en estadística descriptiva en el entorno de mercado general y específico. Se pretende establecer la percepción de la gente con respecto al tema. Haciendo de este estudio una aplicación de la estadística descriptiva para generar lineamientos estadísticos más que parámetros de industria

Palabras Claves: Etnocompetitividad, Textiles Ancestrales, Pluriculturalidad, Cultura, Diversificación.

\section{Introducción.}

Los textiles ancestrales son concepciones místicas que unen el pasado y el presente, a su vez representan alternativas de crecimiento y diversificación textil, permitiendo la innovación progresiva y la presentación de nuevos productos, este enfoque potencia una industria que de por sí ya maneja varios millones de dólares y tiene varios seguidores perceptivos en el mundo. (Morín, Ramirez Suarez, Navarro Garza, \& Estrella Chulim, 2008)

Las etnias y culturas a través de la historia han hecho varias acciones para tratar de diferenciarse, con el tiempo la vestimenta fue uno de los signos distintivos más representativos dando nacimiento al término de aculturación, el mencionado término en el entorno contemporáneo moderno indica que una cultura, su ideología, y sobre todo su idiosincrasia se ha plasmado en un signo objetivo como es la vestimenta. (Brines Tyrer, 1988)

En el país en sus inicios los textiles, fibras y tejidos solían ser primitivos, hasta la llegada de los españoles en la Conquista donde al combinar el conocimiento del nuevo mundo con la fuerza laboral del nuevo, nació una industria que con el tiempo se volvería más y más competitiva, los referentes a nivel textil de la época, estuvieron ubicados de forma inespecífica sin embargo algunos de estos territorios sobresalieron con el tiempo resaltando Otavalo, Peguche y Cotacachi. (Valarezo \& Torres, 2004)

Las metodologías manuales quedaron en el olvido por la captación instantánea de los aborígenes, la nueva tecnología no se hizo esperar con la introducción de telares a pedal y los tornos de hilar, indicando que desde sus inicios la innovación de la industria crece a pasos agigantados. (Cuvi, 2011)

Anteriormente en el país, los textiles y tejidos solían ser respetados en la sociedad así como todos los grupos relacionales a estos, su función era la de delimitar social y económicamente a las personas, dando a denotar que las diferencias estaban muy marcadas en una sociedad que en un principio se creía primitiva y con el tiempo se ha demostrado que en ciertos aspectos no lo era. (Vivanco, 2015)

La producción textil evolucionó, concentrando en las poblaciones más importantes y en los centros urbanos cercanos a los mismos. En el siglo 16 con la instauración de la Real 
audiencia de Quito la industria textil tuvo un crecimiento y perfeccionamiento específico, tan es así que con los años se entiende que la economía primitiva o el eje económico de la época se centraba precisamente en esta actividad. (Ibarra, 2007)

La industria doméstica y su pequeño mercado tuvieron una evaluación que fue desde el tratamiento de lana hasta la confección en algodón, a mediados del siglo veinte, específicamente en la década comercial de 1950 se afianzó la utilización de la llamada fibra. (Arellano, 2014).

La diversificación de materiales se ha dado desde mediados del siglo 20 hasta las primeras décadas del siglo 21, en la cual se hace referencia a la utilización de poliéster, nylon, seda y varios materiales sintéticos derivados del caucho y del plástico.

(De Vidas , 2002)

Desde los años 50 la evolución de la industria textil ha sido muy grande generando empleos para miles de personas y convirtiéndose en un sector complementario en el país.

Es indispensable entender que el sector textil en el país no siempre fue como se lo conoce, el mercado doméstico casi siempre estuvo presente en las décadas contemporáneas, a pesar de esto su desarrollo e impulso no se dio hasta la década de los 90, donde en sus últimos años con problemas en el país, el esquema ideológico permitió una competitividad parcial y un desarrollo percepcional de la industria. Considerando este entorno es bueno precisar que el crecimiento real de la industria textil se da por una inversión a largo plazo en maquinaria, capacitación y una gestión enfocada a la eficiencia y productividad. (Del Valle, 2007)

El término de competitividad moderna se ha visto rezagada no solo porque los costos y la economía en general no permiten diferenciarse, peor aún tener una ventaja competitiva, sino también porque no existe innovación en los diseños, el consumidor en general no se siente identificado con aspectos que trasciendan a la moda o a la cultura.

En el país la distribución de la industria y el grupo de mayor producción se encuentra ubicado en las provincias de Pichincha, Azuay, Imbabura, Guayas y Tungurahua. Esta última cuenta más de 397 talleres pero apenas representa el 5\% de la producción nacional. (Alencastro \& Ortega , 2016)

El mercado en si tiene lugares pequeños de especialización, como es el caso de Bolívar que representa el $1.6 \%$, indicando que los segmentos específicos de mercado tienen una rentabilidad bastante grande. Aparte se puede saber que la mencionada industria es parcialmente innovadora indicando que pequeños sectores y poblaciones pueden ser competitivos. (Dirección de Inteligencia Comercial e inversiones , 2012)

El Sector textil, es un puntal estratégico en el país, ya que a más de generar ingresos macroeconómicos considerables cercanos a 1300 millones de dólares, esta industria genera cerca de 174000 puestos, sin considerar el resto de la industria manufacturera. (León, 2017). 
El gobierno ha considerado al sector como un puntal para la economía estatal, considerando que su diversificación relacionada llega alrededor de 33 ramas, aparte que al no ser un sector muy nuevo, tiene la capacidad de producir con calidad y contribuir a la expansión de mercados relacionales. (El Comercio , 2017)

Esta industria y el mercado en general suele funcionar con telas planas y telas de punto, estas a su vez han permitido una participación segmentado de artículos referenciados principalmente con: Camisetas, polos, jeans, ropa interior, etc. (PRO ECUADOR, 2014).

Entre estas esta la diversificación a través del legado cultural de los pueblos que con el tiempo están siendo aceptada a través de varios targets, que al sumarlos se obtiene la identidad como país. (CEPAL, Población Indígna Ecuatoriana, 2014).

Los textiles ancestrales son de mucha valía cultural, y hasta económica, indicando que una mujer indígena con indumentaria promedio puede tener un costos aproximado de mil dólares, la industria en si ve la limitación de producción no solo por el factor mencionado, sino también porque la empresa ecuatoriana tiene carencia de materias primas, una alta carga impositiva, y aparte el proteccionismo gubernamental ha hecho que se cree una economía burbuja que a la larga le quita competitividad en la región.

Esto con el tiempo ha creado un sentir en el empresario Ecuatoriano, el cual trata de diversificar sus productos y sus líneas para con el fin de crear un crecimiento continuo y poder hacer una sustitución de importaciones. (Alencastro \& Ortega , 2016)

Según la Constitución Política del Ecuador en su artículo 21, que indica: Que las personas están en plena libertad de construir y mantener la identidad cultural, decidir a que cultura pertenecen y a expresarlo, a conocer profundamente el patrimonio cultural de las mismas. (UNESCO, 2008).

Las leyes en la nación estan direccionadas al respeto de los derechos indivudales y colectivos, sin embargo el proceso de aculturización se ha vuelto tan fuerte que la identidad ancestral y cultural de varios pueblos se pierde en el tiempo.

El proceso de aculturización es un proceso contemporáneo en el que gente sin identidad cultural sólida, adapta rasgos característicos primarios o secundarios de una o varias culturas extrajeras. Cada día sucede un proceso de aculturización especialmente en países tercer mundistas que creen que la pobreza y problemas propios del sistema económico están dados por causas ideológicas culturales. (Arellano, 2014).

El contexto global actual están muy relacionadas a grupos de moda con géneros contemporáneos o modernistas, como el Pop, el Rock o suburbanismos marcados como la 
cultura Emo, indicando que sus seguidores no son estudiosos de la filosofía que los creo sino más bien adoptadores tempranos de signos primarios y secundarios que con el pasar del tiempo se han modificado a los extremismos sociales. (Hannerz, 2014)

El Ecuador en la actualidad se empieza asemejar mucho a la sociedad norteamericana, que a lo largo de su historia empezó adoptar personas de varios países del mundo y a través de su mezcla se creó una identidad que aún no ha sido definida.

Los textiles ancestrales han sido estudiados desde varios enfoques haciendo referencia a la valía del tema:

Tabla 1: Los textiles ancestrales han sido estudiados desde varios enfoques.

\begin{tabular}{|c|c|c|c|}
\hline AÑo & AUTOR & ARTICULO & PARAFRASEO \\
\hline 2002 & $\begin{array}{l}\text { Blenda Femenias } \\
\text { University of } \\
\text { Wisconsin - } \\
\text { MadisonJ Tehrani, } \\
\text { M Collard Journal of } \\
\text { Anthropological } \\
\text { Archaeology, 2002 - } \\
\text { Elsevier }\end{array}$ & $\begin{array}{c}\text { COLONIAL } \\
\text { ECUADORIAN } \\
\text { YNDIAS AND THEIR } \\
\text { TEXTILES }\end{array}$ & $\begin{array}{l}\text { Esta investigación se centra en Quito como un centro urbano colonial y próspero en la } \\
\text { producción textilera en el siglo XVI, pues pese a la colonización española sus actividades } \\
\text { estaban social y económicamente fortalecidas. La metodología utiliza una recopilación de } \\
\text { archivos notariales de Quito y el análisis descriptivo del textil de trece indígenas y una mestiza } \\
\text { registrados en testamentos de } 1588 \text { a } 1609 \text {. El resultado permite inferir que a través del textil se } \\
\text { tejieron diversas tramas de índole económico, social y de género en Quito derivados de } \\
\text { diversos patrones culturales. }\end{array}$ \\
\hline 2008 & $\begin{array}{c}\text { Halperin, Christina } \\
\text { T. Ancient } \\
\text { Mesoamerica; } \\
\text { Cambridge Tomo } \\
\text { 19, N. 1, (Spring } \\
\text { 2008): } 111-125 .\end{array}$ & $\begin{array}{l}\text { CLASSIC MAYA } \\
\text { TEXTILE } \\
\text { PRODUCTION: } \\
\text { INSIGHTS FROM } \\
\text { MOTUL DE SAN } \\
\text { JOSÉ, PETEN, } \\
\text { GUATEMALA }\end{array}$ & $\begin{array}{l}\text { El textil como constructo social desde la antigua Mesopotamia denota un estatus y filiación } \\
\text { social como símbolos de pertenencia. Por ello la investigación refererida se fundamenta en } \\
\text { una revision de piezas arqueológicas sobre la producción textil e infiere a partir de su } \\
\text { construcción procesos del tejido. El método aplicado sigue una revisión de las economías } \\
\text { mayas de los hogares respecto a la producción del textil en el Motul de San José. La } \\
\text { investigación plantea como resultado que la producción textil empleada para tributo era una } \\
\text { asunto de interés en las élites pues contaban con los recursos necesarios para su producción. }\end{array}$ \\
\hline 2008 & $\begin{array}{c}\text { Anne M Giangiulio, } \\
\text { University of Texas } \\
\text { at El Paso } 2008\end{array}$ & $\begin{array}{l}\text { In the Weave: } \\
\text { Bhutanese } \\
\text { Textiles and } \\
\text { National } \\
\text { Identity' Exhibit } \\
\text { logo and panels }\end{array}$ & $\begin{array}{l}\text { El arte de la producción textil, rico en técnicas y cultura, habla de las vivencias históricas de los } \\
\text { pueblos y agrega valor a las diferentes generaciones culturales. El arduo trabajo, el empeño, el } \\
\text { amor a su cultura manifestada por sus creadoras enriquece una tarea que apasiona y transmite } \\
\text { belleza a los textiles. }\end{array}$ \\
\hline 2012 & $\begin{array}{l}\text { Robyn Maxwell - } \\
2012\end{array}$ & $\begin{array}{l}\text { Textiles of } \\
\text { Southeast Asia: } \\
\text { Trade, tradition } \\
\text { and } \\
\text { transformation }\end{array}$ & $\begin{array}{l}\text { Los textiles como agentes antropológicos e históricos son portadores de la cultura de un país. A } \\
\text { través de ellos se pueden establecer una serie de significaciones y simbolismos. Evocan } \\
\text { tradiciones y técnicas peculiares e instrínsecas de cada región. La obra analiza los textiles del } \\
\text { sudeste asiático y se centra en la relación entre las tradiciones de pueblos originarios y las } \\
\text { influencias que se deriban del fenómeno de la globalización. Como resultado se arroja la } \\
\text { documentación del proceso como un recurso detallado de procesos tradicionales y nuevos } \\
\text { procesos a los que llegaron los artesanos por el flujo ingresante de la glocalización. }\end{array}$ \\
\hline
\end{tabular}

Elaborado por: Grupo de Investigación.

Materiales y métodos. 
Los textiles ancestrales representan una tendencia no muy explotada en el entorno, representa una opción de diversificación relacionada en la industria textil y una opción de diversificación no relacionada para la industria del turismo y temas relacionados con la cultura.

El presente estudio se enmarca explícitamente en la Provincia de Tungurahua como foco para la zona centro en la cual se enmarca un estudio con el 95\% de confianza, un error que asciende al $5 \%$.

Las probabilidades al ser un estudio de carácter mayormente exploratorio queda con 0.5 correspondiente a la variabilidad de los datos y resultados, para lo cual el tamaño muestral resultante asciende a 382 encuestas.

Los puntos evaluados en las herramientas de investigación son:

Percepción de la existencia, percepción adquisición, parámetros de oferta, mercado actual, frecuencia potencial de compra, medios, factores de compra, percepción en prendas de vestir, percepción en prendas ancestrales.

La investigación se ha centrado con la cuantificación de variables nominales y ordinales que son consideradas importantes en la evaluación del mercado.

\section{Resultados.}

Gráfica 1: Conocimiento del término y entorno de textiles ancestrales.

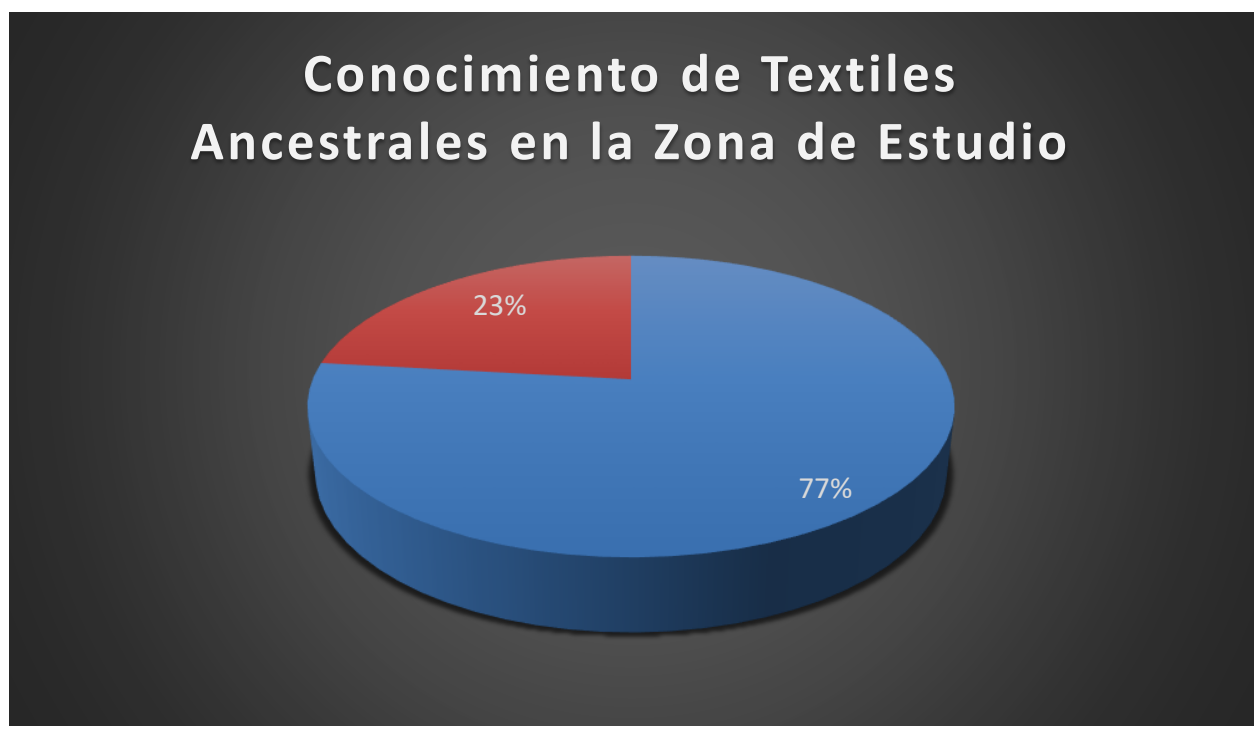

Elaborado por: Grupo de Investigación.

Gráfica 2: Aceptación del producto 


\section{Aceptación de compra textiles Ancestrales}

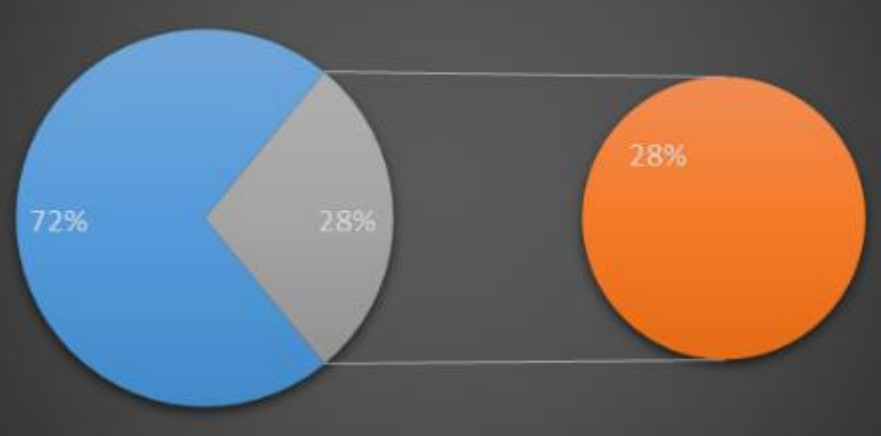

Elaborado por: Grupo de Investigación.

Gráfica 3: Conocimiento de proveedores - Oferta en el mercado actual.

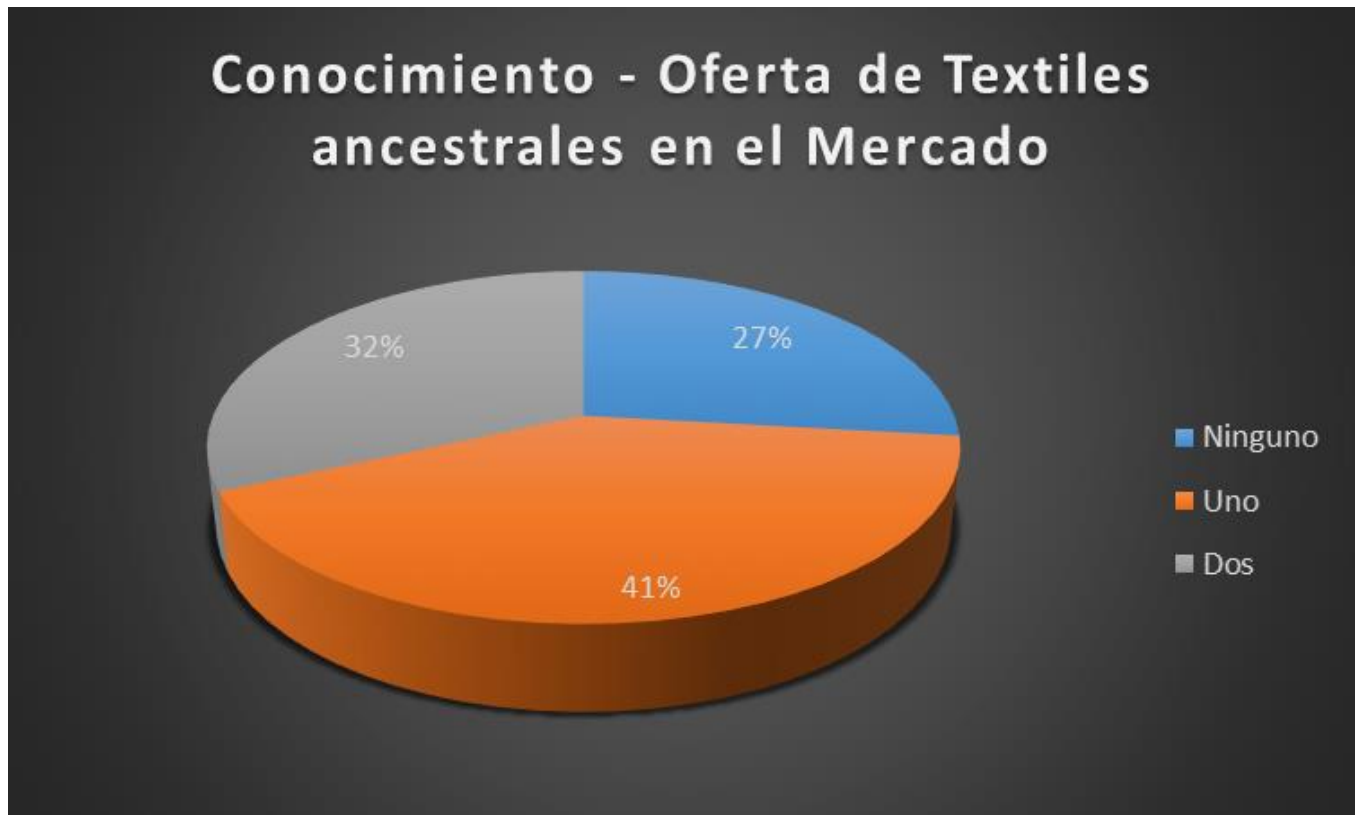

Elaborado por: Grupo de Investigación.

Gráfica 4: Satisfacción con los textiles adquiridos. 


\section{Satisfacción - Calidad por textiles en el mercado}

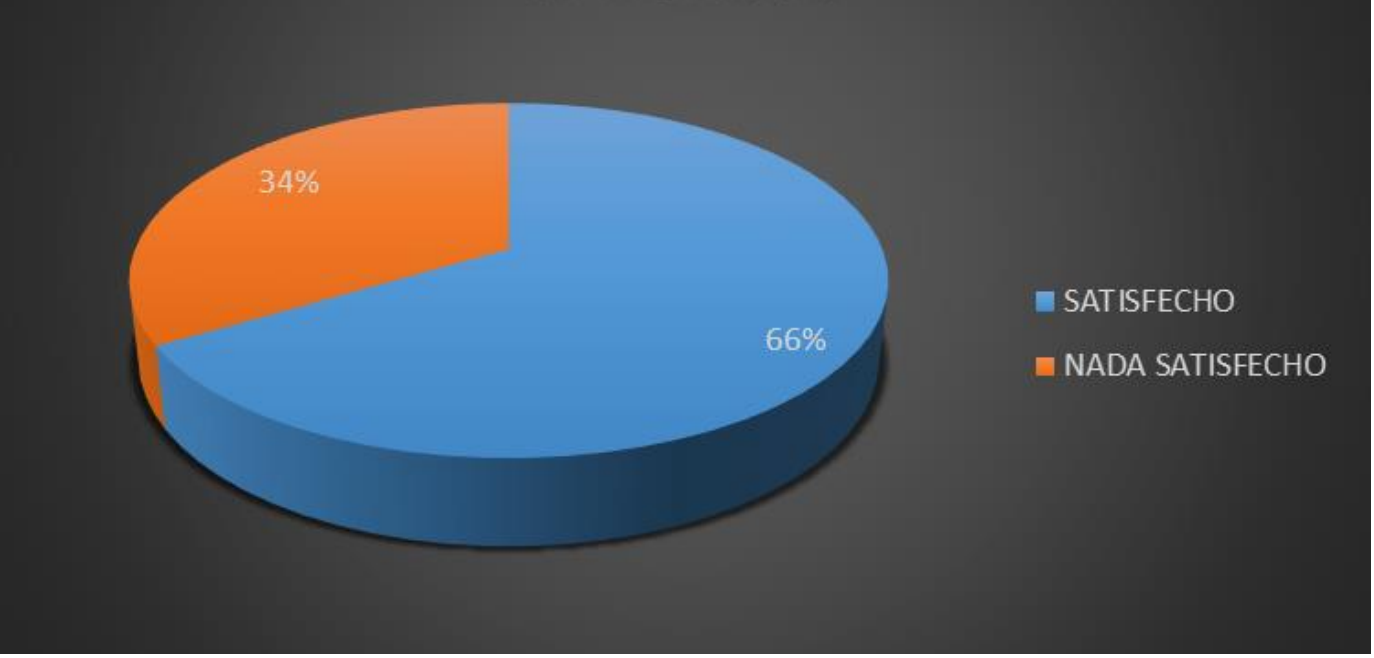

Elaborado por: Grupo de Investigación.

Gráfica 5: Frecuencia de Compra en Textiles Ancestrales.

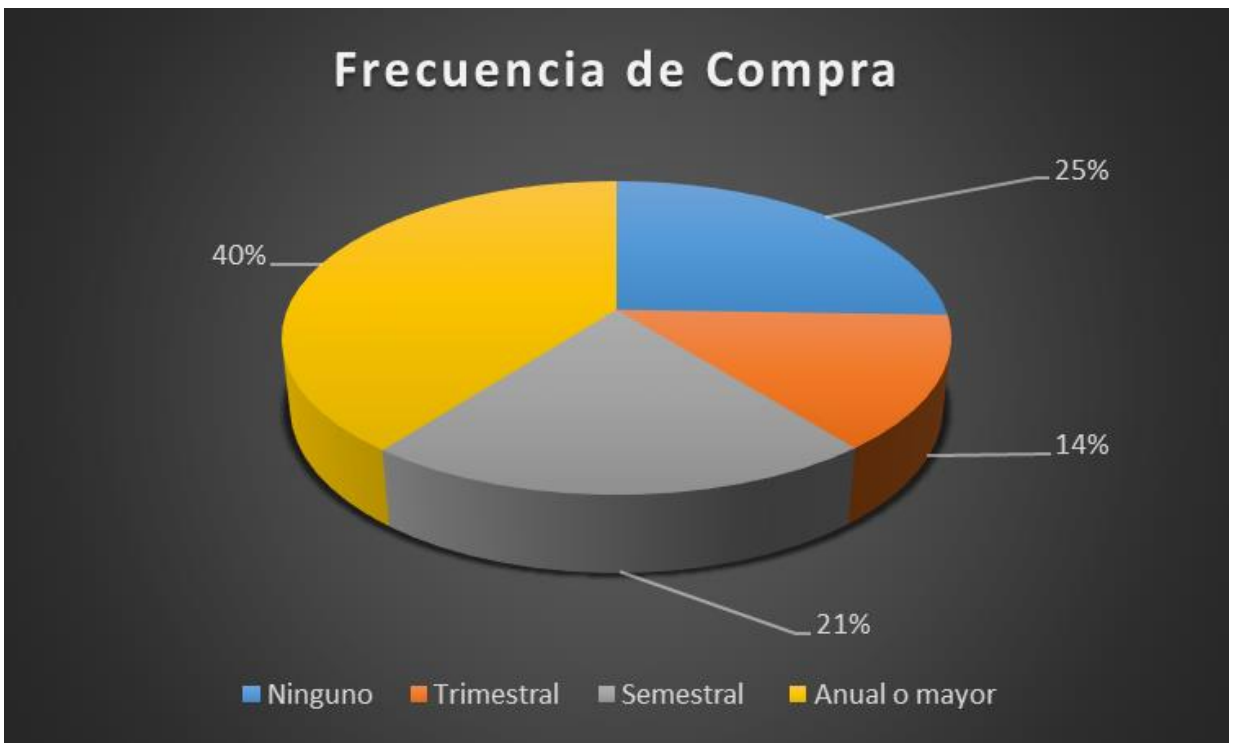

Elaborado por: Grupo de Investigación.

Gráfica 6: Medios de difusión y promoción para la industria textil. 


\section{Medios de Difusión y promoción en} la industria de textiles ancestrales

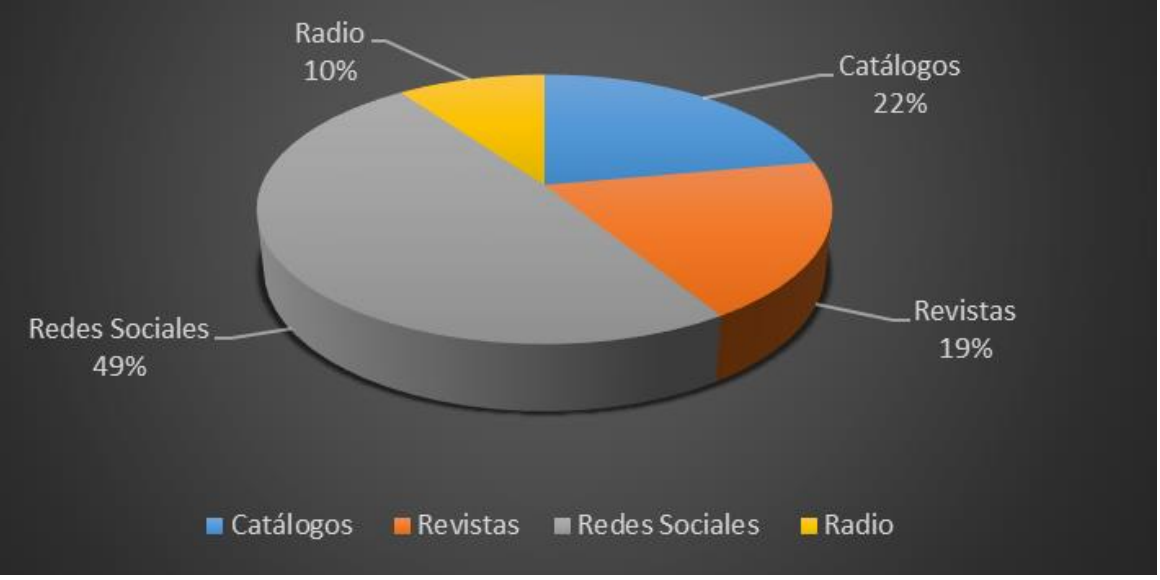

Elaborado por: Grupo de Investigación.

Gráfica 7: Factores primarios de consumo en la industria textil

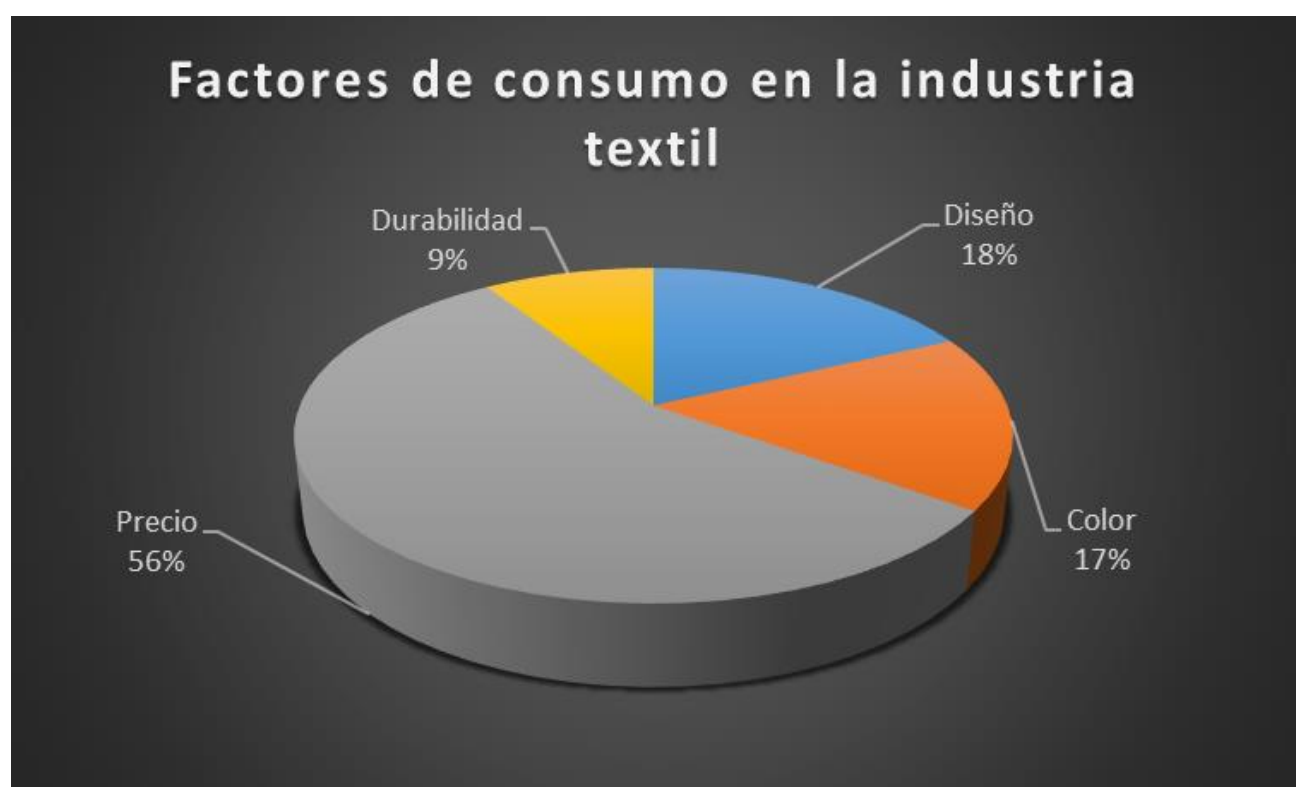

Elaborado por: Grupo de Investigación.

Gráfica 8: Factores de consumo primarios específicos para textiles ancestrales. 


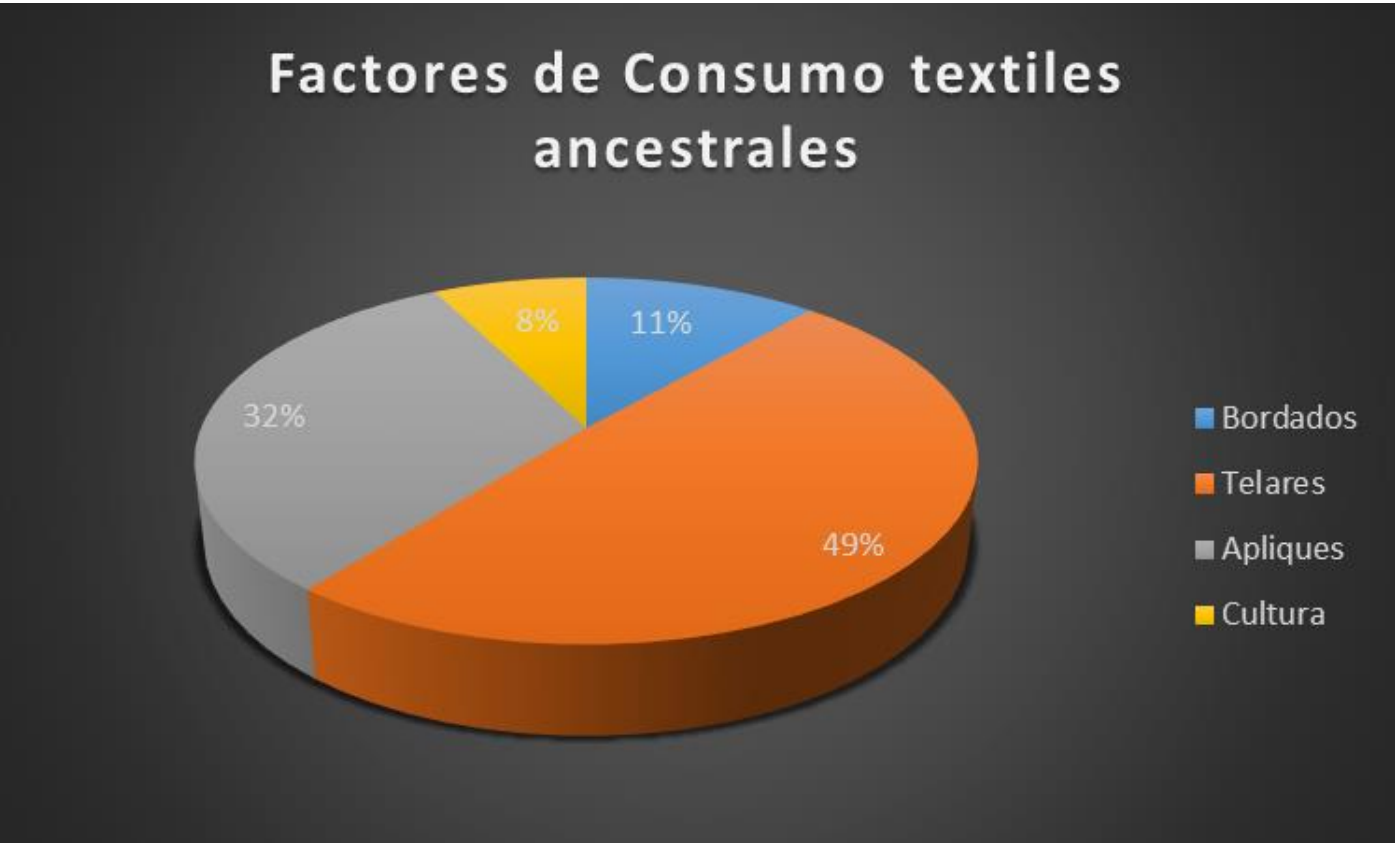

Elaborado por: Grupo de Investigación

\section{Conclusiones.}

- En el estudio se puede observar que la gente no tiene un conocimiento absoluto de lo que representan los textiles ancestrales, sin embargo el término en si representa mucho en la percepción del usuario. La frecuencia relativa de conocimiento de los textiles asciende al $77 \%$.

- A pesar de que la población estudiada no conoce varios de los factores influyentes en los textiles ancestrales del total de personas representativas en la investigación solo el $72 \%$ está de acuerdo con la calidad existente en el mercado de textiles. Se observa un target que no es mayormente entendido, sin embargo la presión para la oferta y la calidad de productos es explicita.

- Se puede entender que la industria, y en si la oferta no es mayormente evolucionada en el país por que se indica que el $41 \%$ de los encuestados solo conoce un proveedor de estos textiles, el $32 \%$ conoce al menos dos, y el $27 \%$ menciona que no conoce ninguno.

- Del total de encuestados, haciendo referencia los textiles ancestrales, y productos culturizados en la industria textil, el $66 \%$ se encuentra satisfecho con la calidad encontrada en el mercado.

- La frecuencia de compra mayormente se la hace anual, con una frecuencia relativa del $40 \%$, entendiendo que no son productos ligados a una necesidad básica, sino que más bien están regidos por una especialización vinculada a un deseo.

- El mercado textil es un mercado de evolución contemporánea rápida donde las redes sociales en difusión publicitaria y comercio han llegado a representar cerca del $40 \%$. Entre los factores más representativos al momento de comprar un producto de 
la industria textil se destaca el precio como factor referencial y el diseño, por ser uno de los factores más fuertes a nivel percepcional.

- En los textiles ancestrales, se puede observar que el factor con mayor peso al momento de realizar una compra es la calidad y estructura del telar.

\section{Referencias bibliográficas.}

AITE. (2010). Una de cal y otra de arena. Quito: AITE, Asociación de Industriales Textiles del Ecuador.

AITE. (2011). Publicaciones y Estudios. Quito: Asociación de la Industria Textil Ecuatoriana.

AITE. (2013). Publicaciones y Estudios período 2008 - 2013. Quito: Asociación de la

Alencastro, C. P. E., \& Ortega, R. A. S. (2016). ANÁLISIS DEL SECTOR TEXTIL ECUATORIANO 2009-2013. Revista Científica ECOCIENCIA, 3(4).

Amaya, J. (2012). Gerencia: Planeación y Estrategia. Madrid-España: Santo Thomás.

American Psychological Association. (2012). Publication manual ++-of the American Psychological Association. Scopus.Washington, DC: American Psychological Assoc.

Anderson, Charles \& Johnson (2003). The impressive psychology paper. Chicago: Lucerne Publishing

Arciniega, P.R; \& Alarcón, Ch.J (2016). Indigenous Peoples and Poverty: Ecological Wealth versus Social Poverty. Contradictions and prospects for development in Latin America. SCIELO. Buenos Aires-Argentina. Print versión ISSN 009100046. Consejo Nacional de Investigaciones Científicas y Técnicas http://dx.doi.org/11.1692/S010671872016000200008 .

Arellano, J.G. (10 de octubre de 2014). Identidad Cultural en Ecuador. Obtenido de www.elcomunicadorpucesi.blogspot.com/2012/03/la-identidad-cultural-es-parte-dela.html: http://elcomunicadorpucesi.blogspot.com/2012/03/la-identidad-cultural-esparte-de-la.html.

Artal Castell, M. (2015). Dirección de ventas. Madrid: ESIC.

Baca Urbina, G. (2012). Evaluación del Proyecto de Inversión. México D.F.: Paraninfo S.A.

Benalcazar, M.G. (2013). Activos a Nivel Empresarial. México D.F.: Ansure CS.A. 
Bertucci, H; Pereira (2016) et al. Tourism and urbanization: environmental problems of The araruama Lagoon. SCIELO. Estate of Rio de Janeiro, Brazil. Ambient. soc., Dec 2016, vol.19, no.4, p.59-80. ISSN 1414-753X.

Bouchard, G., La región culturelle: un concept tríos objects. Essays de mise au point, en Fernad Harvey (ed.), Scopus. La región culturelle, Institut québécois de recherche sur culture, Québec, 1989

Brines Tyrer, R. (1988). Historia demográfica y económica de la Audiencia de Quito: población indígena e industria textil 1600-1800. Quito: Banco Central del Ecuador.

Brysk, A (2012). From Tribal Village to Global Village: Indian Rights and International Relations in Latin América. Scopus. Stanford University Press

Bustos, P; Amigo, H; Muños, S; \& Martorrel, R (2015). Growth in indigenous and nonindigenous Chilean School children from 3 Poverty Strata. American Journal of Public Health: SCIELO. Print versión ISSN 00900036. Consejo Nacional de Investigaciones Científicas y Técnicas http://dx.doi.org/11.1592/S010571872012000200006 .

Calegare, M.G; Aguilar, H.M.I; \& Gasparetto, B.A (2014). Povos e comunidades tradicionais: das áreas protegidas à visibilidade política de grupos sociais portadores de identidad étnica e coletiva. SCIELO Ambient. soc., Set 2014, vol.17, no.3, p.115134. ISSN 1414-753X

Cardini, L.A (2012). Producción Artesanal Indígena: Saberes y Prácticas de los Qom en la ciudad de Rosario. SCIELO. Print versión ISSN 01047183. Consejo Nacional de Investigaciones Científicas y Técnicas-Argentina http://dx.doi.org/10.1590/S010471832012000200005 .

Carranco, S.G. (2012). INEC: Fascículo Provincial de Tungurahua. Quito-Ecuador: INEC.

Carrasco, F.S. (20 de junio de 2017). Secretaria del Buen Vivir. Obtenido de www.secretariabuenvivir.gob.ec/el-buen-vivir-en-la-constitucion-del-ecuador/: http://www.secretariabuenvivir.gob.ec

Case, K; \& Fair, R. (2012). Principios de Microeconomía. México D.F.: Prentice Hall Hispanoamérica S.A.

CEPAL. (12 de Diciembre de 2014). Obtenido de www.CEPAL.org

CEPAL. (2014). Población Indígena Ecuatoriana. Quito-Ecuador: Ministerio de Gobierno www.CEPAL.org.

COMEX. (2009). Resolución N 466. Salvaguardias. Quito: Comité de Comercio Exterior. 
Constitución Política de la República del Ecuador. (2008). Registro Oficial 449. Montecristi, Ecuador.

Cuvi, N. (2011). Auge y decadencia de la fábrica de hilados y tejidos de algodón La Industrial, 1935-1999. Procesos. Revista ecuatoriana de historia, 1(33), 63-95.

De Vidas, A. A. (2002). Memoria textil e industria del recuerdo en los Andes: identidades a prueba del turismo en Perú, Bolivia y Ecuador. Editorial Abya Yala.

Del Valle, M. (2007). Tejiendo capacidades para la exportación. Textiles y confecciones del Ecuador y el mercado de los EEUU. CAF.

Domínguez, R.T. (2013). Financiamiento Empresarial. México D.F.: Arquero S.A.

Doyle, M., Fryer, T. B., \& Cere, R. (2010). Éxito Comercial. Prácticas administrativas y contextos culturales. Boston: Cengage Learning.

Ecuavisa. (22 de Septiembre de 2014). Ecuavisa. Obtenido de http://www.ecuavisa.com/articulo/noticias/internacionales/81183-83-poblacionamerica-latina-indigena-segun-reciente-informe.

Esteban, M; \& Nadal, J.M . (2012). La Construcción Narrativa de la Identidad a través del Conflicto y la Ventrilocuación. Revista Digital SCIELO, 45.

Garcés, T.A.C. (2016). Diseño de Bocetos de la Empresa O--Glan. Ambato-Ecuador: O-Glan.

García, D.F. (19 de junio de 2016). Empresa O--Glan. Obtenido de www.google.com.ec/maps/place/1\%C2\%B014'45.0\%22S+78\%C2\%B037'01.8\%22W /@-1.2451253,-78.6178051,18z/data=!4m5!3m4!1s0x0:0x0!8m2!3d-1.245845!4d78.617159 https://www.google.com.ec/maps/place/1\%C2\%B014'45.0\%22S+78\%C2\%B037'01.8 $\% 22 \mathrm{~W} / @-1.2451253,-78.6178051,18 \mathrm{z} / \mathrm{data}=! 4 \mathrm{~m} 5 ! 3 \mathrm{~m} 4$ !1s0x0:0x0!8m2!3d$1.245845 ! 4 \mathrm{~d}-78.617159$

Gavilanes, J (2017). Nacionalidades y Pueblos del Ecuador: Salasaca. Scopus http://gruposetnicosecu.blogspot.com/2017/04/salasaca.html.Scopus

Gómez, C.O.R. (2014). Las Inversiones a NIvel Empresarial. Revista Académica Con el Número Internacional Normalizado , 45.

Gonzalez, L. (2015). Caracterización del Subsentor. Ambato- Ecuador: PUCESA .

Hannerz, U. (2014). Cosmopolitas y locales en la cultura global. Alteridades, (3), 107-115. 
Hora, L. (11 de Enero de 2011). La Hora. Obtenido de http://lahora.com.ec/index.php/noticias/show/1101087270/-

1/LA_IDENTIDAD_IND\%C3\%8DGENA_FRENTE_A_OTRAS_RAZAS.html\#.V7 zX2LjhDIU Klotler, P. \& Armstrong, G. (2013). Fundamentos de Marketing México D.F.: Cengage Learning Editores, S.A.

http://www.scielo.br/scielo.php?script=sci_subject\&lng=es\#subj6.

Ibarra, H. (2007). Los estudios sobre la historia de la clase trabajadora en el Ecuador.

Industria Textil Ecuatoriana.

INEC. (2010). Infoeconomía. Quito: Instituto Nacional de Estadísticas y Censos.

INEC. (2012). Infoeconomía. Quito: Instituto Nacional de Estadísticas y Censos.

INEI. (2013). Exportación FOB de productos no tradicionales, 20

INEXMODA. (2012). Observatorio económico nacional del sistema de moda. Plataforma de Innovación. Colombia: Instituto INEXMODA.

Klotler, P. \& Armstrong, G. (2013). Fundamentals of Marketing. México D.F.: Cengage Learning Editores, S.A.

Koop, E.R; \& Zelin, B (2016). New Fashion Areas for Designing Apparel through the Flan Pattern. USA: Fashion Institute of technologies.

Krugman, P. R. (2012). Economía Internacional. México: Siglo XXI.

Lamb, C.W,; Jair,J.F.; \& McDaniel. (2012). Marketing Empresarial. México D.F.: Cengage Learning Editores S.A.

León, I (2015). El Textil Artesanal: Los Telares. SCIELO rin versión ISSN 01067185. Consejo Nacional de Investigaciones Científicas y Técnicas-Argentina http://dx.doi.org/10.1690/S0109-71832012000200009

Lugo-Morin, D. R., Ramírez-Juárez, J., Navarro-Garza, H., \& Estrella-Chulim, N. G. (2008). Etnocompetitividad del sistema artesanal textil Mitla, el papel del territorio y la innovación. Economía, sociedad y territorio, 8(28), 981-1006.

Manjarrez, G.C. (2012). Activos Fijos Contables. México D.F.: Antillas S.A.

Matriz Productiva del Ecuador. (2017). Perfil del Consumidor a Prendas Textiles Dening. Quito-Pichincha: Ministerio del Gobierno. 
Mattei, M.M.C. 2013. "Endangered Indigenous Languages of Venezuela: New Governmental Policy and Projects of Revitalization: the particular case of two Carib languages, Mapoyo and Yawarana" en O. Scopus. Sakyana (ed.) Lectures on endangered Languages: 2 from Kyoto Conference 2000. Kyoto: ELPR. 315-333.

Molinillo, L.S. (2014). Distribución Comercial Aplicada . Madrid-España: SCIELO.

Nuñes, P.T. (22 de 10 de 2016). Activo corriente. Obtenido de http://knoow.net/es/cieeconcom/contabilidad/activo-corriente/:

http://knoow.net/es/cieeconcom/

Pontificia Universidad Católica del Ecuador. (15 de 10 de 2008). UNESCO. Obtenido de www.unesco.org/culture/natlaws/media/pdf/ecuador/ecuador_constitucionpo_08_spa orof:

http://www.unesco.org/culture/natlaws/media/pdf/ecuador/ecuador_constitucionpo_0 8_spaorof

Pro Ecuador. (2012). Índice de Productividad Textil nacional. Comercial e Inversiones . Dirección de Inteligencia. Quito-Ecuador: Instituto de promoción y de Exportaciones.

ProEecuador. (9 de 10 de 2014). Dirección de Inteligencia Comercial e Inversiones: Dirección de Promoción de Exportaciones. Obtenido de http://www.proecuador.gob.ec/wpcontent/uploads/2013/11/PROEC_AS2012_TEXTILES.pdf: http://www.proecuador.gob.ec

Rendón Monzón, J. J. (2003), La comunidad. Modo de vida de los pueblos indios. México: Dirección General de Culturas Populares e Indígenas. SCIELO

Salasaca-Runa kuna. (20 de Junio de 2009). Salasaca-Runa kuna. Obtenido de http://salasaka-runakuna.blogspot.com/2009_06_20_archive.html

Salcedo, E. (2014). Moda Ética para un futuro Sostenible. Barcelona - España: Editorial Gustavo Gili.

Salvador, M.H. (2012). Mercadotecnia Programada. México D.F.: Limusa.

Salvador, P.F. (2012). Canales de Distribución . México. D.F.: Paraninfo S.A.

Sampieri, R. H., Collado Fernández, C., \& Baptista Lucio, P. (2003). Metodología de la Investigación. México, D. F: McGraw-Hill.

Sancho, V.F. (04 de 07 de 2017). PLan de inversiones. Obtenido de www.inversion-es: http://www.inversion-es.com/plan-de-inversiones.html 
Santillana S. A. (2012). Los primeros pobladores de nuestro territorio (12000 a. C-550 a. a.). Quito: Santillana.

Secretaria de Educación Superior. (2013). El Dialogo de los Saberes Ancestrales en los Estados Plurinacionales. En C. C. Imbaquingo, El Dialogo de los Saberes Ancestrales en los estados Plurinacionales (María Eugenia Paz y Miño ed., pág. 7). Quito. Obtenido de www.educacionsuperior.gob.ec.

Secretaria del Buen Vivir. Obtenido de http://www.secretariabuenvivir.gob.ec/el-buenvivir-en-la- constitución-del-ecuador/ 57

SENPLADES. (2009). Plan Nacional para el Buen Vivir 2009 - 2013. Quito: Secretaría Nacional de Planificación y Desarrollo.

SENPLADES. (2013. Plan Nacional para el Buen Vivir 2013 - 2017. Quito: Secretaría Nacional de Planificación y Desarrollo.

Stavenhagen, R. (1965), "Clases, Colonialism and Acculturation", en Studies Comparative International Development, núms. 4-7. SCIELO. Rin versión ISSN 01077195. Consejo Nacional de Investigaciones Científicas y Técnicas-Argentina http://dx.doi.org/10.1690/S0109-71832012000200009

Stern, L. Ansary, A; \& Coughlan, A. (2012). Canales de comercialización. Madrid- España: Prentice Hall.

Valarezo, G. R., \& Torres, V. H. (2004). El desarrollo local en el Ecuador: historia, actores y métodos. Editorial Abya Yala.

Velasco, V.C. (2012). La Industriaa Textil Ecuatoriana". Quito-Ecuador: Ministerio de Gobierno.

Verduga, P.T. (2014). Presupuesto de costos. Chile: Antillas S.A.

Vivanco, G. (2015). Historia Industria Textil-Ecuador. Taller de Diseño.

Wheeler, S; \& Hirsh, E. (2012). Distribution Channels. Bogota-Colombia: Norma S.A.

Zambrano, A. (2012). Planificación Estratégica Presupuesto y Control de la Gestión Pública. Caracas- Venezuela: IGEZ S.A.

Para citar el artículo indexado. 
Cobo P., Mancheno J., Guamán C. \& Mancheno M. . (2018). Etnocompetitividad textil, tendencia histórica que marca el futuro empresarial. Revista electrónica Ciencia Digital 2(3), 318-334. Recuperado desde: http://cienciadigital.org/revistacienciadigital2/index.php/CienciaDigital/article/view/160/14 $\underline{0}$

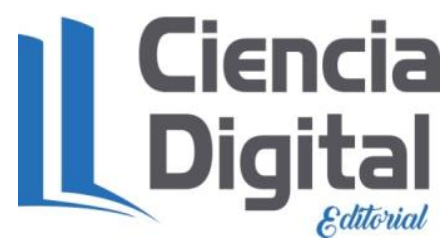

El artículo que se publica es de exclusiva responsabilidad de los autores y no necesariamente reflejan el pensamiento de la Revista Ciencia Digital.

El articulo queda en propiedad de la revista y, por tanto, su publicación parcial y/o total en otro medio tiene que ser autorizado por el director de la Revista Ciencia Digital.
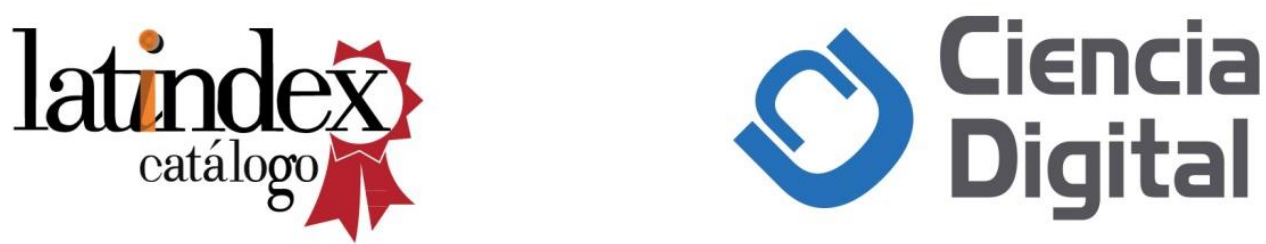